\title{
Performance and Evaluation of Adaptive Dynamic Channel Allocation protocol in Hybrid Multichannel Wireless Mesh Networks
}

\author{
Shaji.K.A.Theodore, \\ Dept of IT, Al Musanna College of Technology
}

\author{
P.Sumi Dolly Fidelis, \\ Dept of ETC, Al Musanna College of Technology
}

\begin{abstract}
Today, there are various methods available for increasing the throughput of a multichannel wireless mesh network. These can be either static allocation or dynamic allocation. In this study, a hybrid multichannel wireless mesh networking architecture is proposed and every mesh node has both static and dynamic interfaces. Static-Dynamic Combined Channel Allocation protocol (SCCA) is analgorithm proposed, considering both throughput and delay in the channel assignment. SCCA gives advantages of both static and dynamic allocation methods. Interference and Congestion Free Routing (ICFR) is included to further improve the throughput of the wireless mesh network. Simulation results indicate that ICFR decreases the packet delay considerably without affecting the network throughput.
\end{abstract}

\section{General Terms}

Wireless mesh network, hybrid channel allocation, multichannel, routing.

\section{INTRODUCTION}

Wireless Mesh Networks is one of the upcoming technology, used in most of the applications. Even though it is used widely, faces the problem of capacity reduction due to interference. This interference problem can be avoided by using multiradio routers. Channel allocation to the interfaces in multiradio router is a major issue, which increases the network capacity.

There are two channel allocation methods. In static allocation, channel is fixed with interface of the mesh router. Whereas, in dynamic allocation, interface is allowed to change the channel whenever required. Static channel allocation gives less overhead but it depends on the constant traffic in the network. Centralized channel assignment proposed in [1], [2] needs the exact traffic pattern. Whereas in [3], [4] make use of statistical traffic, which is already known. But dynamic channel allocation [5], [6] having more overhead due to channel switching. Dynamic channel allocation is preferred whenever there is change in the traffic pattern. Two channel allocation methods having their both advantages and disadvantages. A hybrid architecture is proposed in this paper, which combines the advantages of both methods. In this architecture, dynamic channel allocation method is used by one of the interface in each router, while the other interfaces use the static channel allocation method. The links working on dynamic channel enhances the network connectivity and the network's adaptivity to the changing traffic while the links working on static channels provide high throughput paths from end-users to the gateway. Therefore, this hybrid architecture provides better adaptivity compared to the purely static architecture without much increase of overhead compared to the purely dynamic architecture.
This paper discusses several issues in the hybrid wireless mesh network. 1) The system architecture discussing the coordination of the channel assignment between static and dynamic interfaces, hence the channel resources could be utilized efficiently. 2) Dynamic interface Channel Allocation: Static-Dynamic Combined Channel Allocation protocol (SCCA) is proposed, which considers both throughput and delay in the channel assignment. Compared with MMAC [6], SCCA is able to reduce the packet delay without reducing the network throughput. 3) Routing: in the hybrid structure, we have static links and dynamic links, both of which can be used to transmit data. We propose an Interference and Congestion Free Routing protocol (ICFR), which aims at balancing the channel usage over the network and thus improve the network throughput. The paper is organized as follows: Previous works are discussed in Section 2. Introduction of network model in Section 3. In Sections 4 gives the proposed algorithm for channel allocation. In section 5, the routing algorithm is presented. Evaluation of the protocols in Section 6 , and finally conclusion in Section 7.

\section{RELATED WORK}

WMN is used in various applications [7]. Interference leads to the capacity reduction is the issue faced by the multihop wireless networks. [8], [9] studies the interference effect on the same channel and [10] studies the effect of overlapping channels. [11] studies the interference and throughput of single-radio single-channel wireless network. Raniwala et al. [1] proposed an iterative approach to satisfy all traffic profiles, removing the joint routing and channel assignment problem. [2] and [12], describes the problem using linear programming, classified as NP hard. It proposed algorithms to get a channel assignment and routing scheme. Raniwala and Chiueh [3] constructed load balancing tree from the network topology, Assigning channels to the links using load-aware algorithm considering the major traffic of internet gateways to the clients. In [4], the peer-to-peer traffic was assumed to be dominant. Using original network topology to construct a kconnected backbone, and then assigned channels on the constructed topology and minimized interference by assigning channels to constructed topology.

[5], [6], [14], [15] proposes link level dynamic channel allocation strategies, having frequent channel switching among the communicating nodes. Overhead by switching and coordination mechanism are the issues to consider. Dynamic channel assignment methods can be categorized into two approaches. 1) Dedicating one interface from each mesh node for the use of control only [5]. This method does not require synchronization among nodes but resources are not used efficiently. 2) No interface is dedicated for control. Hence, resources can be used efficiently, but this approach needs synchronization among nodes [6], [14], [15]. 
[13], [16], [17] proposes some dynamic channel allocation algorithms, having less frequent channel switch. In [13], a central server recalculates the channel assignment for the whole network when there is a change in the environment, and conveys the nodes to change the channel. Pediaditaki et al. [16] proposes a distributed routing and channel allocation algorithm for the network, where each node has only two radios. [17] proposes a approach where nodes autonomously able to learn their channel allocation from the information of neighborhood channel usage. Whenever there is change in the traffic pattern, this learning algorithm needs time to converge to a good channel assignment. In this paper, dynamic interfaces uses link level channel allocation algorithms.

Unlike previous approaches, this paper propose a hybrid architecture, in each mesh node one of the interfaces will be using dynamic channel allocation method and static channel allocation method on the other interfaces. This hybrid wireless mesh network is going to have the advantages of both channel allocation methods. [18] proposed a hybrid multichannel allocation protocol (HMCP). In each node, some interfaces works with fixed channel while the remaining interfaces to change the channels. When the dynamic interface of one node switches to the same channel with the static interface of the other node, the data transmission takes place.HMCP having high delay due to multihop data transmission, because the channel switch is required on each node. But, the proposed hybrid architecture allows static interfaces to transmit data to other static interfaces. Therefore, proposed approach achieves lower delay than HMCP.

[19] studies wireless interference in directional antenna networks. [20] and [21] proposed channel assignment algorithms using multiple orthogonal channels to increase the network throughput. Dynamic channel switching creates overhead in directional antennae, hence, these algorithms use static assignment strategies. Cost-efficient and widely used omni-directional antenna networks has been focused in this paper. Routing metrics need to be designed to improve the throughput of WMNs. [22] provided a comparison of different routing metrics used in static multihop wireless networks. [23] proposed a new metric Weighted Cumulative ETT (WCETT) which is more efficient than other traditional metrics in multiradio multichannel WMNs. This paper focusing on the routing in the hybrid architecture containing both static and dynamic links. To improve the total throughput in the network, Interference and Congestion Free routing protocol is proposed.

\section{NETWORK MODEL}

This paper proposed to use the hybrid architecture to achieve low channel switching overhead and adaptivity to changing traffic. Considering the network topology as $\mathrm{G}(\mathrm{V}, \mathrm{E})$ where $\mathrm{V}$ represents the mesh routers and pairs of mesh routers is represented by $\mathrm{E}$, within communication range. Assuming each mesh router has multiple interfaces, we allow only one interface of each mesh router to switch channel frequently, called as static interface and fixed channels in the remaining interfaces, called as dynamic interface.

Fig. 1 is a hybrid multichannel wireless mesh network. Most of the mesh nodes have 3 interfaces, and some boundary nodes $(r, v, n, u)$ have 2 interfaces. In each mesh node, one of the interface will be dynamic and others interface will be static

Aim of the static interface is to maximize the throughput from end-users to gateways, which is a major portion of the traffic in the network. [3] proposed some heuristic algorithms, could be used. In this algorithm, a load balanced tree is constructed for each gateway. The tree is used to allocate bandwidth to each user according to the throughput. Each link is assigned channel after the topology construction. High priority is given to the links closer to the gateways to be allocated with less congested channels. In Fig. 1, bold lines are called as static links. Static links Channel allocation is shown in the figure.

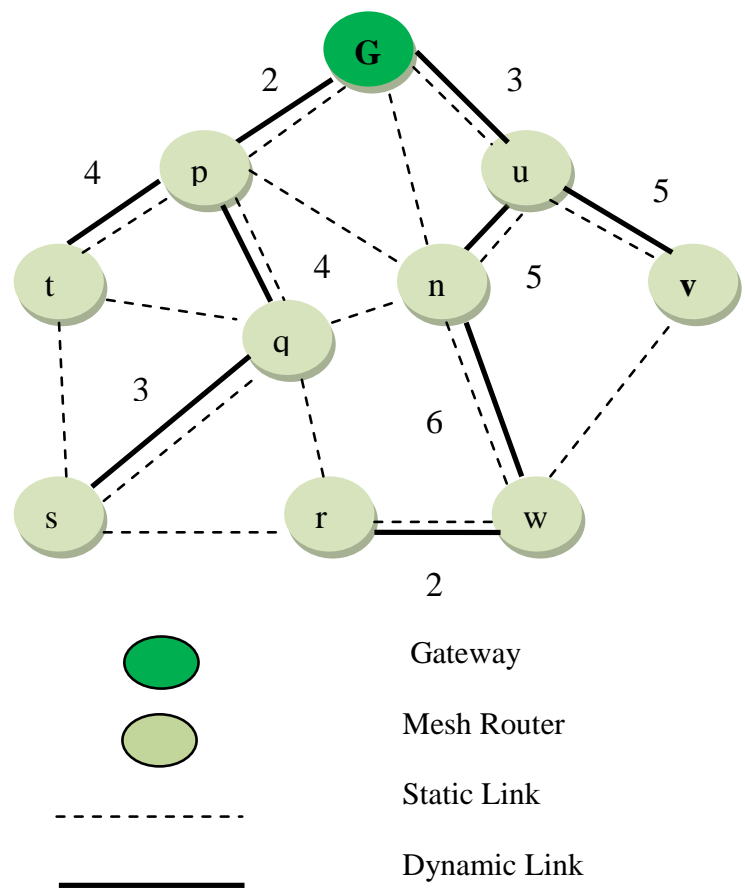

Fig. 1 The hybrid WMN architecture

Two dynamic interfaces able to negotiate a common channel when they are in transmission range of each other. Communication takes place when they have data to transmit. These links are called as dynamic links. Fig. 1 shows all possible dynamic links in dotted lines. Dotted line stresses that the only pair of nodes can communicate. TDMA is used here. Time is divided into fixed-length intervals. Each interval consists of control and data interval. During control interval, all nodes communicate using a default channel to negotiate the channels to be used in the data interval. Nodes transmit and receive data on the negotiated channels during the data interval. Care should be taken in the dynamic channel allocation to avoid interference from the static interfaces.

In control interval, using default channel each dynamic interfaces negotiate. Interference from the static interfaces must be eliminated during the control interval. Negotiation default channel is not used in the channel allocation of the static interfaces. This will not affect the efficiency of channel usage, because dynamic interfaces can still use this default channel in the data interval.

Low congested channel is chosen as the data channel. In control interval, each dynamic interface communicates with neighborhood using default channel, aware of the channel usage of dynamic interfaces. Channel usage of the static interfaces cannot be determined in the same way. In this situation, static nature of wireless mesh networks can be used to solve the hidden terminal problem. Usually wireless mesh networks have static topology, each mesh node maintains a stable list of other mesh nodes within its interference range. By allowing each mesh node to measure the channel usage of its static interface and can send to all other mesh nodes that 
are within their interference range. Hence, the channel usage of the static interfaces are known to dynamic interfaces within its interference range.

Advantages of this hybrid architecture are as follows:

In pure static channel allocation method, the connectivity of the network topology may be degraded, leads to suboptimal routing paths. But using dynamic interface, the connectivity of the topology is well maintained since it is able to communicate with any other interface within radio transmission range.

Pure static channel allocation methods cannot adapt to the frequent change in the network traffic. However, dynamic links is able to direct traffic around the congested areas and therefore achieve better load balance in the network. In order to simply the protocol design, let that dynamic and static interfaces will not communicate with each other.

\section{STATIC-DYNAMIC COMBINED CHANNEL ALLOCATION}

Multiple channels in wireless mesh networks can increase the throughput. But, there is a tradeoff between throughput and delay. The proposed channel allocation protocol, considers both network throughput and packet delay, while adapting to changing traffic. Following are the design of the StaticDynamic Combined Channel Allocation algorithm.

MMAC [6] compared with single channel MAC protocol 802.11, doubles the throughput. MMAC improves throughput but packet delay increases. In MMAC, before transmitting data, every two nodes switches to the same channel in each time interval, and thus, in one interval, each packet can be transmitted at most one hop away. MMAC considers only throughput not the delay. This, leads to the design of StaticDynamic channel allocation protocol (SCCA). SCCA achieve lower packet delay satisfying the traffic needs.

MMAC may cause unnecessary packet delay when traffic load is in below saturation, which is shown by examples in Fig. 2. In Fig. 2, Consider A has some data to be transmitted to $C$. By MMAC, during $\mathrm{t} 1$ interval, packets are transmitted from A to B and in 2 interval, packets are transmitted from B to $\mathrm{C}$. So, two intervals are required to transmit. However, one interval is enough to transmit packet from A to $\mathrm{C}$.

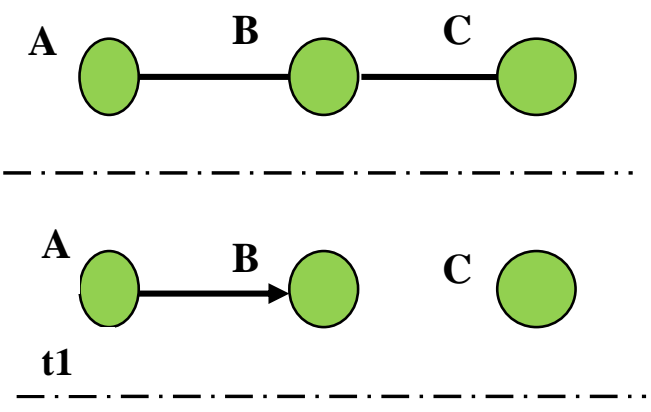

A<smiles>C1CCCCC1</smiles>

$\mathbf{t 2}$

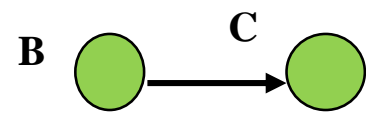

Fig 2. MMAC Delay
MMAC allows only a pair of nodes to negotiate common channel in each interval which causes delay as described in above case. Delay can be reduced by allowing more than two nodes to negotiate a common channel in each interval. If A, B, and $\mathrm{C}$ negotiate a common channel together, only one interval is required to complete all the transmissions, which reduces the delay.

SCCA divides time into fixed length intervals. Each interval is further divided into control interval and data interval. Let $\mathrm{T}$ be the interval length, and Tc, Td be the length of control interval and data interval, respectively. During the control interval, all the nodes switch to default channel and negotiate channels. During the data interval, the nodes transmit and receive data among each other using negotiated channel. In SCCA, T is set to $100 \mathrm{~ms}$, and Tc is set to $20 \mathrm{~ms}$.

Every dynamic interface has multiple queues in the link layer. This queue is used for buffering the data to transmit. SCCA is similar to MMAC, in the first step of channel negotiation. If a dynamic interface has some data to transmit, it selects a neighbor and tries to negotiate a common channel. Queue length is the criteria for the selection of neighbor and duration of time, for which it has not been served. Finally, two nodes negotiated common channel as shown in Fig. 3a.

SCCA is different from MMAC, which enables further channel negotiation among nodes. After the completion of channel negotiation between the two nodes, the node that initiated negotiation called as sending node, and the other one is called as receiving node. Another node type, called as pending node, which is not succeeded in negotiating a channel with any other node. Algorithm 1 describes the channel negotiation process. Whether to perform further channel negotiation is determined by the queue length threshold QT. The channel negotiation will not be useful when the queue length is over the threshold.

Algorithm 1.Channel Negotiation

Pending Node:

1: Neighbors are notified that it is a pending node by broadcasting a PENDING_NODE_REQ message.

2: if receiving SWITCH_CHANNEL then

3: As indicated in the message, switch to channel c.

4: end if

Sending Node:

1: if the receiving node has queue length less than QT then

2: Neighbors are notified that it is a sending node by broadcasting SENDING_NODE_REQ the traffic load is below saturation.

3: end if

4: if receiving SWITCH_CHANNEL then

5: if its receiving node $(r)$ is not negotiating with any other sending nodes then

6: As indicated in the message, Switch to channel c.

7: Send Notification to $r$, to switch to channel c.

8: end if

9: end if

Receiving node: 
1: if the receiving node has queue length less than QT then

\section{2: if receiving PENDING_NODE_REQ then}

3: Send SWITCH_CHANNEL message to the pending node including its own channel c.

4: end if

\section{5: if receiving SENDING_NODE_REQ then}

6: Send SWITCH_CHANNEL message to the sending node including its own channel $\mathrm{c}$.

\section{7: end if}

8: end if

Fig.3.a shows the MMAC working. In Fig 4.b shows how the SCCA works. There are three cases.

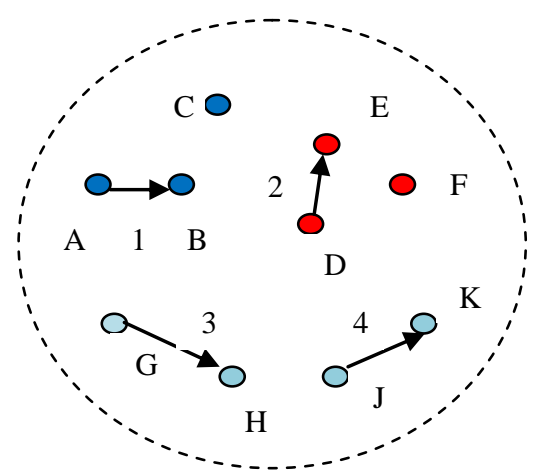

Fig. 3a- SCCA

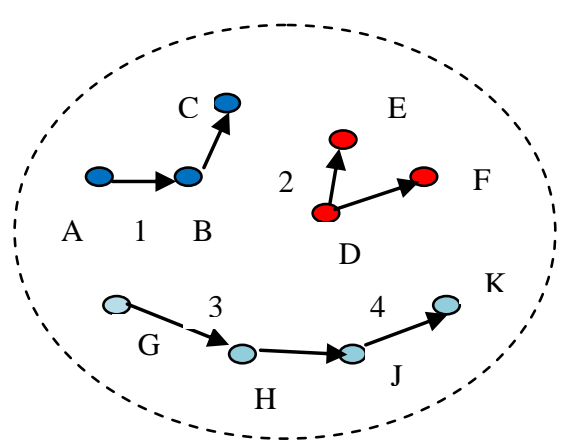

Fig.3b- SCCA

The example in Fig. 3b shows the working of our protocol.

I) Node $\mathrm{A}$ has some data to $\mathrm{C}$, where $\mathrm{A}$ and $\mathrm{B}$ can transmit in the common channel. So in this cases B negotiate with $\mathrm{C}$ to work in the same channel as A and B. II) D wants to send data to both $\mathrm{E}$ and $\mathrm{F}$. D and $\mathrm{E}$ having common channel, while $\mathrm{F}$ does not. Hence, D negotiate with $F$ so that $F$ works on the same channel as D and E. III) $G$ wants to send data to $J$ through $\mathrm{H}$. $\mathrm{G}$ and $\mathrm{H}$ are working in the same channel, at the same time $\mathrm{J}$ and $\mathrm{K}$ works on the common channel. Therefore, $\mathrm{H}$ can negotiate with $\mathrm{J}$ so that $\mathrm{G}, \mathrm{H}, \mathrm{J}$, and $\mathrm{K}$ work on the same channel. SCCA can able to negotiate common channels among more than two nodes in each interval when network traffic is not saturated. As a result, SCCA reduce packet delay while satisfying the imposing traffic.

\section{INTERFERENCE AND CONGESTION FREE ROUTING}

To maximize the total throughput in the hybrid network using both static links and dynamic links, the routes of different flows to be selected efficiently avoiding congestion and channel usages are balanced at each node. Following is the proposed interference and congestion free routing metric.

In Fig. 4, A to $\mathrm{C}$ shown in bold lines is the routing path for the flow, with the rate $r$. Interfering wireless links are plotted in gray lines. The flow will consume some bandwidth $r$ on the link AB. However, three links will interfere with $A B$. The flow will consume the same bandwidth in the interfering links. The same thing happens in the link BC. Flow consuming bandwidth of multiple links in the network. But, the flow is using two wireless links.
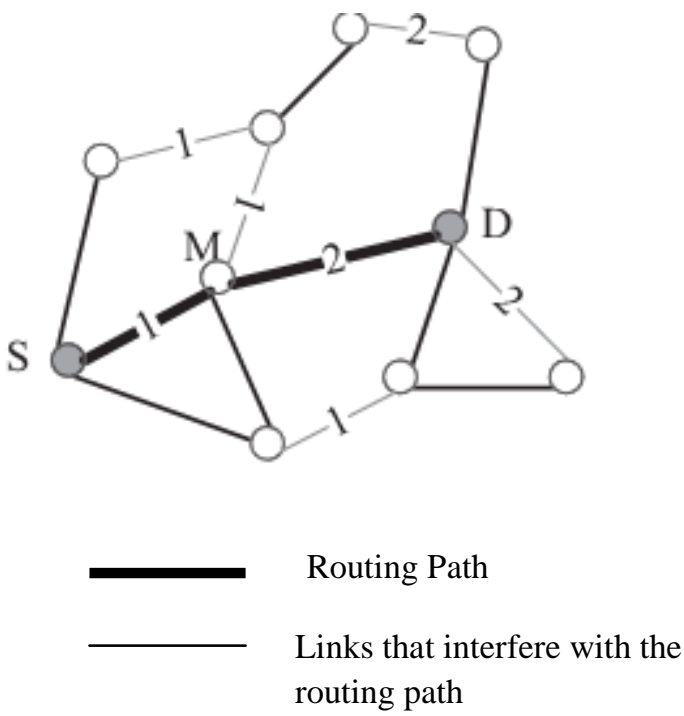

Fig. 4 Effect of interference

Consider $\mathrm{P}$ as the routing path of a flow with rate $\mathrm{r}$. For every link $l \in \square \mathrm{P}$, let IE $(l)$ be the set of links which interfere with 1 . Link 1 has the expected transmission count ETX $(l)$. Formula for calculating the total bandwidth consumed by the flow is as follows.

$$
\mathrm{B}_{\text {total }}=\sum_{l \in \mathrm{P}}|\mathrm{IE}(l)| * \operatorname{ETX}(l) * \mathrm{r},
$$

Comparing to the previous metrics, this metric helps better channel usage. To make the routing better by avoiding congested links, some modifications on Btotal formula is required. Dividing each link into three states as HIGH, MEDIUM and LOW. HIGH means the channel is highly congested, and therefore it is not safe to route traffic through this link. MEDIUM means the channel used by the link has a some amount of load, still can route traffic. LOW means the channel has very light load, and can be used for routing additional traffic. Hence, w1, w2, w3 are the weights for these 3 states. Therefore, they must satisfy w1 $>$ w $2>$ w3. Inducing the weights into $\mathrm{B}_{\text {total }}$, in the path $\mathrm{P}$, the cost of routing a flow of rate $r$ can be calculated as follows:

$$
\operatorname{Cost}_{\text {total }}=\sum_{l \in \mathrm{P}}\left[\operatorname{ETX}(l) * \mathrm{r} * \sum_{\mathrm{e} \in \mathrm{IE}(I)} \quad \mathrm{w}(\mathrm{e})\right]
$$

Therefore, the cost metric of every link as $\left.\mathrm{C}(l)=\operatorname{ETX}(l) * \mathrm{r}^{*} \sum_{e \in I E(I)} \quad \mathrm{w}(\mathrm{e})\right]$ and then use source routing to find a minimum cost path for each flow, $\mathrm{P}^{*}=\operatorname{argmin} \sum_{l \in P} \mathrm{C}(l)$. 
The link states are identified from the queue length. If the length of the queue is more, the link is congested. It is not possible to say that if one of the links in a pair is dynamic, whether they will interfere with each other or not. The other way is to estimation of probability of interference.

Every dynamic links maintains statistics of its channel usage history, in the form of $\quad\{$ pi $\mid i=1,2,3, \ldots C\}$, where pi is the fraction of time during which, the link worked on channel i. This representation can also be used for static links. For example $\mathrm{pc}=1$ and $\mathrm{pi}=0(\mathrm{i} \neq \mathrm{c})$ for the static link works on channel c. 11 and 12 are the two given links, whose channel usage is represented by $\{$ pill $\}$ and $\{$ pil 2$\}$, can predict their probability of interference using the following formula.

$$
\mathrm{P}_{i f}(l 1, l 2)=\sum_{\mathrm{i} \epsilon}(1,2, \ldots \mathrm{C}) \mathrm{pi}^{11} * \mathrm{pi}^{12}
$$

Therefore, the cost formula can be modified as

$$
\operatorname{Cost}_{t o t a l}=\sum_{l \epsilon P}\left[\operatorname{ETX}(l) * r * \sum \mathrm{e}_{\epsilon \mathrm{E}(\mathrm{I})}\left(\mathrm{w}(\mathrm{e}) * \mathrm{P}_{\mathrm{if}}(\mathrm{e}, 1)\right)\right]
$$

It is easy to maintain the channel usage information in the each node. Since, dynamic links change channels in a synchronous fashion. Routing agents are updated periodically about the channel usage information.

\section{PERFORMANCE EVALUATION}

Simulations are performed using NS2 simulator. Q2S patch was used to support multi channels and multiple interfaces per node in the simulator.

Fig. 5 shows the comparison between the SCCA and ICFR protocol. Graph clearly indicates that throughput is increased using ICFR than the SCCA channel allocation algorithm. Even the throughput is increased with SCCA, it is further improved by the interference and congestion free routing.

Packet delay is further reduced by interference and congestion free routing than the SCCA algorithm, which is shown in the Fig.6

Fig.7 shows the Packet Delivery ratio between the ICFR and SCCA. Figure shows that ICFR is better than the SCCA.

\section{CONCLUSION}

In this paper, hybrid wireless mesh network architecture is proposed, where each mesh node has both static and dynamic interfaces. Static-Dynamic Combined Channel allocation protocol is proposed, to be used on dynamic interfaces. Then, Interference and congestion aware routing is used on both static and dynamic links. Compared with SCCA, ICFR reduces the packet delivery delay without degrading the network throughput. In the proposed architecture, static and dynamic interface will not communicate with each other. It can be the future work if the performance is improved considering that situation.

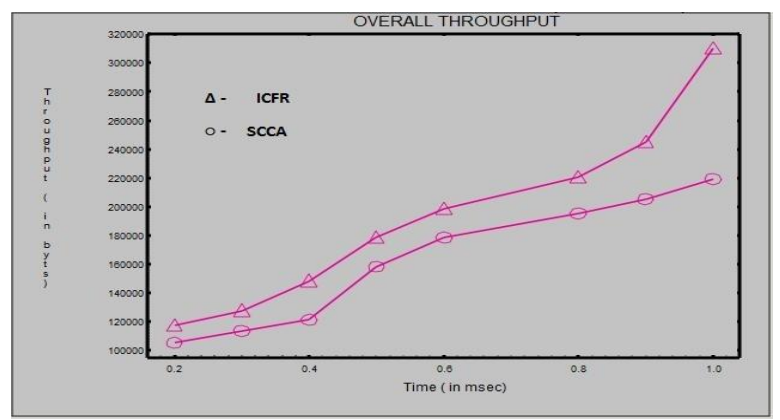

Fig. 5.Throughput

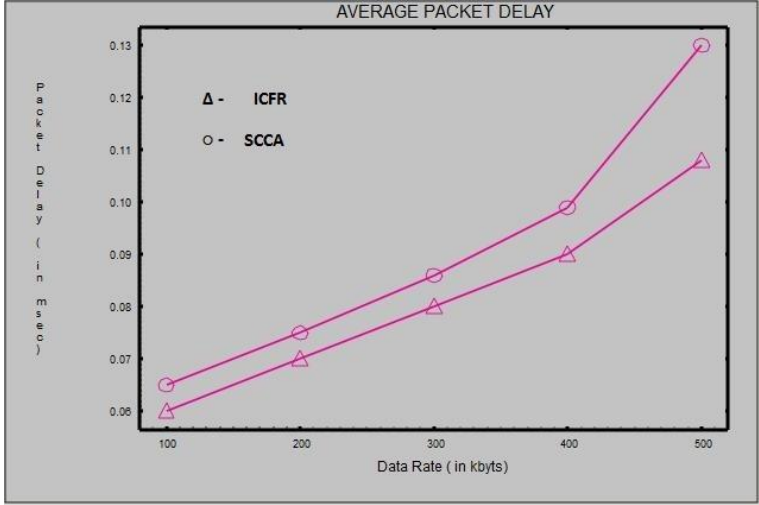

Fig.6.Delay

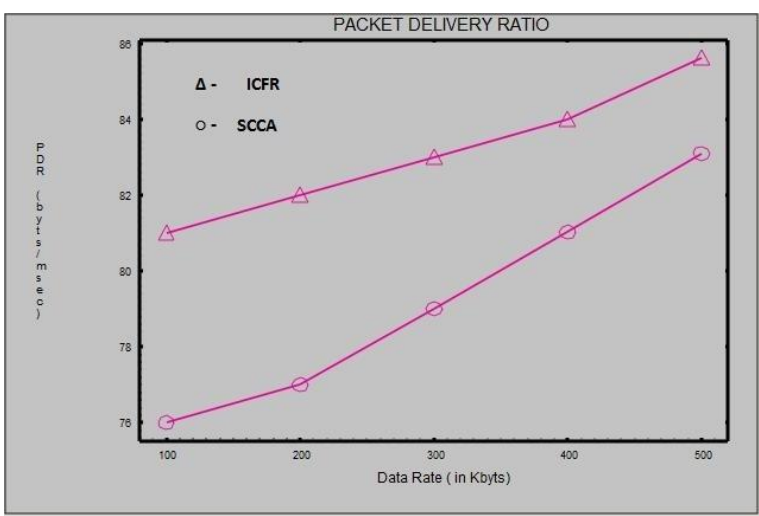

Fig.7 Packet Delivery Ratio

\section{ACKNOWLEDGEMENT}

Our whole hearted thanks to the almighty for guiding us with the knowledge and power for bringing out this publication in an effective manner.

\section{REFERENCES}

[1] A. Raniwala, K. Gopalan, and T. Chiueh, "Centralized Channel Assignment and Routing Algorithms for MultiChannel Wireless Mesh Networks," ACM Mobile Computing and Comm. Rev.vol. 8,pp. 50-65, 2004.

[2] M. Alicherry, R. Bhatia, and L. Li, "Joint Channel Assignment and Routing for Throughput Optimization in Multi-Radio Wireless Mesh Networks," Proc. ACM MobiCom,2005.

[3] A. Raniwala and T. Chiueh, "Architecture and Algorithms for an IEEE 802.11-based Multi-Channel Wireless Mesh Network,” Proc. IEEE INFOCOM, 2005.

[4] J. Tang, G. Xue, and W. Zhang, "Interference-Aware Topology Control and QoS Routing in Multi-Channel Wireless Mesh Networks," Proc. ACM MobiHoc,2005.

[5] S.-L. Wu, C.-Y.Lin, Y.-C.Tseng, and J.-P. Sheu, "A New MultiChannel Mac Protocol with On-Demand Channel Assignment for Multi-Hop Mobile Ad Hoc Networks," Proc. Int'l Symp. Parallel Architectures, Algorithms, and Networks (ISPAN), 2000.

[6] J. So and N. Vaidya, "Multi-Channel Mac for Ad Hoc Networks: Handling Multi-Channel Hidden Terminals Using a Single Transceiver," Proc. ACM MobiHoc,2004. 
[7] I.F. Akyildiz, X. Wang, and W. Wang, "Wireless Mesh Networks: A Survey," Computer Networks, vol. 47, pp. 445-487, 2005.

[8] P. Gupta and P.R. Kumar, "The Capacity of Wireless Networks," IEEE Trans. Information Theory, vol. 46, no. 2, pp. 388-404, Mar. 2000.

[9] J. Padhye, S. Agarwal, V.N. Padmanabhan, L. Qiu, A. Rao, and B. Zill, "Estimation of Link Interference in Static Multi-Hop Wireless Networks, Proc. Internet Measurement Conf.,2005.

[10] M. OE, “Advanced Internet Technology ii: Internet Operation -Wireless Network Operation," http://www.soi.wide.ad.jp/class/20040013/slides/09, 2010 .

[11] K. Jain, J. Padhye, V. Padmanabhan, and L. Qiu, "Impact of Interference on Multi-Hop Wireless Network Performance," Proc. ACM MobiCom,2003.

[12] M. Kodialam and T. Nandagopal, "Characterizing the Capacity Region in Multi-Radio Multi-Channel Wireless Mesh Networks," Proc. ACM MobiCom,2005.

[13] K.N. Ramachandran, E.M. Belding, K.C. Almeroth, and M.M. Buddhikot, "Interference-Aware Channel Assignment in MultiRadio Wireless Mesh Networks," Proc. IEEE INFOCOM,2006.

[14] P. Bahl, R. Chandra, and J. Dunagan, "SSCH: Slotted Seeded Channel Hopping for Capacity Improvement in IEEE 802.11 AdHoc Wireless Networks," Proc. ACM MobiCom,2004.

[15] W.-H. Tam and Y.-C. Tseng, "Joint Multi-Channel Link Layer and Multi-Path Routing Design for Wireless Mesh Networks," Proc. IEEE INFOCOM,2007.
[16] S. Pediaditaki, P. Arrieta, and M.K. Marina, "A Learning-Based Approach for Distributed Multi-Radio Channel Allocation in Wireless Mesh Networks," Proc. IEEE Int'l Conf. Network Protocols (ICNP),2009.

[17] A. Dhananjay, H. Zhang, J. Li, and L. Subramanian, "Practical, Distributed Channel Assignment and Routing in Dual-Radio Mesh Networks," Proc. SIGCOMM,2009.

[18] P. Kyasanur and N. Vaidya, "Routing and Link-Layer Protocols for Multichannel Multi-Interface Ad Hoc Wireless Networks," ACM SIGMOBILE Mobile Computing and Comm. Rev.vol. 10, pp. 31-43, 2006.

[19] B. Raman and K. Chebrolu, "Revisting Mac Design for an 802.11-Based Mesh Network," Proc. HotNetsIII,2004.

[20] B. Raman, "Channel Allocation in 802.11-Based Mesh Networks," Proc. IEEE INFOCOM,2006.

[21] P. Dutta, S. Jaiswal, and R. Rastogi, "Routing and Channel Allocation in Rural Wireless Mesh Networks," Proc. IEEE INFOCOM,2007.

[22] R. Draves, J. Padhye, and B. Zill, "Comparison of Routing Metrics for Static Multi-Hop Wireless Networks," Proc. SIGCOMM,2004.

[23] R. Draves, J. Padhye, and B. Zill, "Routing in MultiRadio MultiHopWirelss Mesh Networks," Proc. ACM MobiCom,2004.

[24] Y. Ding, K. Pongaliur, and L. Xiao, "Channel allocation and Routing in Hybrid multichannelmultiradio Wireless Mesh Networks,' 